\title{
Nerve tissue into mastectomy scar. Its trap may lead to chronic pain?
}

\section{Aris Giannos ${ }^{1}$, Sofoklis Stavros ${ }^{1,2}$, loannis Thanopoulos ${ }^{1}$, Emmanouil Stamatakis ${ }^{3}$, Konstantinos Dimitrakakis ${ }^{1}$, Peter Drakakis ${ }^{1,2}$, Dimitrios Loutradis ${ }^{1}$}

${ }^{1} 1^{\text {st }}$ Department of Obstetrics and Gynecology, "Alexandra" Hospital, National and Kapodistrian University of Athens, Greece

${ }^{2}$ Unit of Reproductive Molecular Biology and Recurrent Abortions and Recurrent Abortions Unit, Assisted Reproduction Division, $1^{\text {st }}$ Department of Obstetrics and Gynecology, "Alexandra" Hospital, National and Kapodistrian University of Athens, Greece

3Department of Anesthesiology, "Alexandra" General Hospital of Athens, Greece

\section{Corresponding Author}

Sofoklis Stavros, MD PhD, Obstetrician and Gynecologist, Fellow of the $1^{\text {st }}$ Department of Obstetrics and Gynecology, National and Kapodistrian University of Athens, General Hospital of Athens "Alexandra", Loulou and Vasilissis Sofias Ave, 11528 Athens, Greece, Tel.: +30 6906725017, e-mail: sfstavrou@yahoo.com

\section{Abstract}

The purpose of the present article is to present a case of a 64-year old woman that underwent mastectomy and presented with severe pain and palpable nodes on the mastectomy scar 2 years following the operation. Excision of the mastectomy scar and histological evaluation revealed nerve branches that were in contact with the muscular tissue and that resided between the fibrous and adipose tissue. Following the second procedure the pain was alleviated.

\section{Introduction}

Like any organ and tissue on human body, breast has a specific nerve supply. The breast receives its innervation from the lateral and anterior cutaneous branches of the second to sixth intercostal nerve and from the supraclavicular nerves. Historically, Cooper in 1840 described mammary branches from the 2nd-6th intercostal nerves, and noticed that the nipple was supplied by branches which lay close to the surface of the gland ${ }^{1}$. Many years later with the improvement in dissecting technique gained from microsurgery, Sarhadi et al. (1996) found that the nipple was innervated by the lateral cuta- neous branch of the 4th intercostal nerve, by two branches, one passing superficial to the gland, and the other through the retromammary space, and by variable lateral and medial additional branches from the 2nd-5th nerves, these branches came to lie superficially and formed a subdermal plexus under the areola $^{2,3}$.

\section{Case presentation}

A 64-year-old woman, gravida1, para 1, was presented to gynecological clinic of our hospital with pathologic findings in mammography. Especially at 
her left breast of the outer upper quadrant, $8.5 \mathrm{~cm}$ from the nipple an uncertain finding with internal micro calcifications was presented, while the ipsilateral lymph nodes were normal. In the right breast scattered fibrous and glandular elements with normal morphology calcifications were noted. Lymph nodes were normal.

The patient underwent a breast ultrasound with the following findings: at 12 hour of the left breast, a small iso-echogenic lesion with dimensions of $3 \mathrm{~mm}$ and normal margins was found. Also, ultrasonography revealed enlarged lymph nodes up to $13 \mathrm{~mm}$ in diameter in both axillaries. Abdominal ultrasound was normal. Her medical history included high blood pressure under medication, coronary disease, dyslipidemia and gastritis. Her surgical history included a coronary angiography $(01 / 2014)$ for myocardial infarction with a stent placement. She was nonsmoker, consuming alcohol only in social occasions without allergies. Her body mass index (BMI) was $30.5 \mathrm{Kgr} / \mathrm{m}^{2}$. She underwent a core biopsy of her left breast on June 2014 and the histological diagnosis was suggestive of a ductal carcinoma grade III. Immunohistochemistry analysis in cellular level showed ER stain positive in $60 \%$ of tumor cells, PR in $2 \%$, Ki-67 stain positive in $2 \%$ while C-erB-2 in $0 \%$ of tumor cells. Surgical treatment was the oncological council's decision for the patient, if her performance status was good enough, owning to the fact that she suffered from a crucial cardiovascular disease and the meantime should be at least a year from the stent placement.

Because of her medical status, the patient did not procced to a surgical procedure, but she started hormonal therapy with letrozole for a year. The thorax CT scan and abdominopelvic CT scan six months later revealed no pathological findings and the bone scan was normal also. A month later, patient underwent left mastectomy and lymphadenectomy according to the agreement of cardiologists. Postoperatively, the patient was followed up in the ward without postoperative complications. The patient was compliant with the therapeutic program which was well tolerated with no significant side effects. Histological examination of surgical specimens revealed a ductal carcinoma grade I of $1 \mathrm{~cm}$ in diameter and two invaded axillary lymph nodes out of twenty-three $(2 / 23)$ on the left side. Hormonal markers were as follow: ER 40\%, PR (-), Ki67(-) and HER2(-). The stage of the malignancy was T1N1M0 based on TNM staging. Oncological council's decision was chemotherapy and hormonal therapy.

The post treatment follow revealed no pathological findings (CT scan of thorax and abdomen, tumor markers Ca 15-3, CEA, Ca 125). Two years later, patient returned complaining for pain and palpable nodes on the mastectomy area. Ultrasound examination of the area showed a cystic collection on the lower part of the mastectomy with dimensions of 6 $\mathrm{x} 4 \mathrm{~mm}$, another cystic collection with dimensions of $15 \times 8 \mathrm{~mm}$ in the surgical trauma of the left axillary and under that a second hypo echogenic nodule approximately $7 \times 6 \mathrm{~mm}$ with normal margins. Due to cardiovascular disease she decided to postpone a second operation for removing the breast scars. Hence, two years later on March 2018 she underwent a second surgical procedure for the palpable lesion's removal. During the operation she removed two parts from the previous mastectomy scar and the three palpable lesions. Postoperative clinical images are illustrated in Figure 1. Histological examination and immunochemistry analysis of surgical specimens revealed that between the fibrous tissue and the adipose tissue, nerve branches in contact with muscular tissue, were observed (Figure 2, 3). This finding and the fact that patient relieved from pain after that operation is the remarkable aspect of this case report.

In total, patient after first surgery referred pain and irritation, symptoms that are usually seen after 

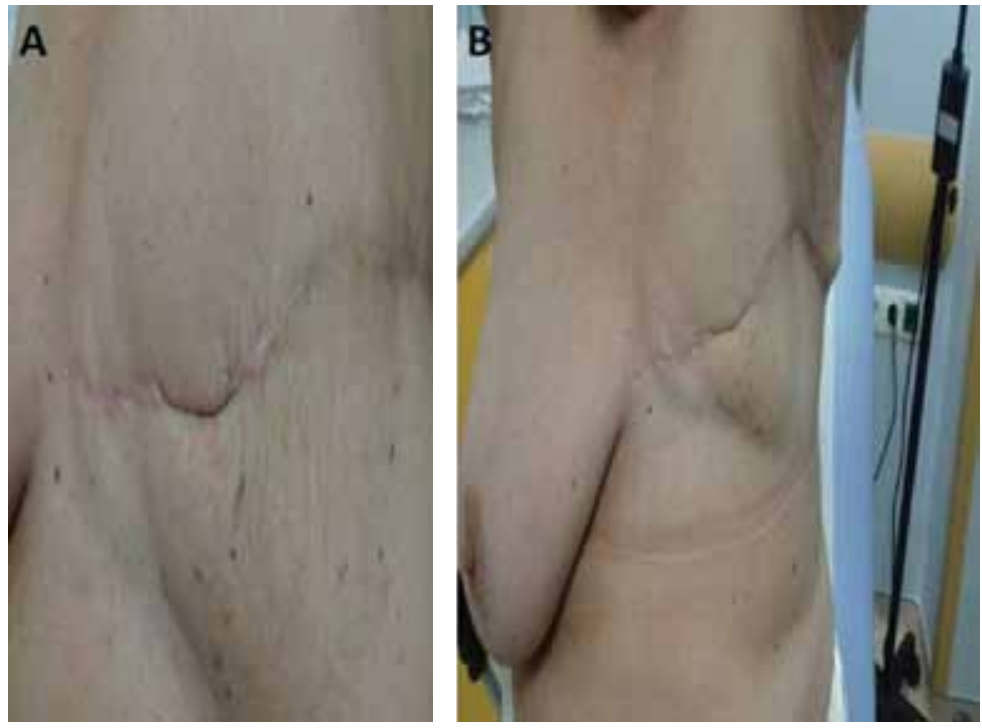

Figure 1. A; B: Clinical images of the left breast after the removal of mastectomy scar and the palpable lesions.
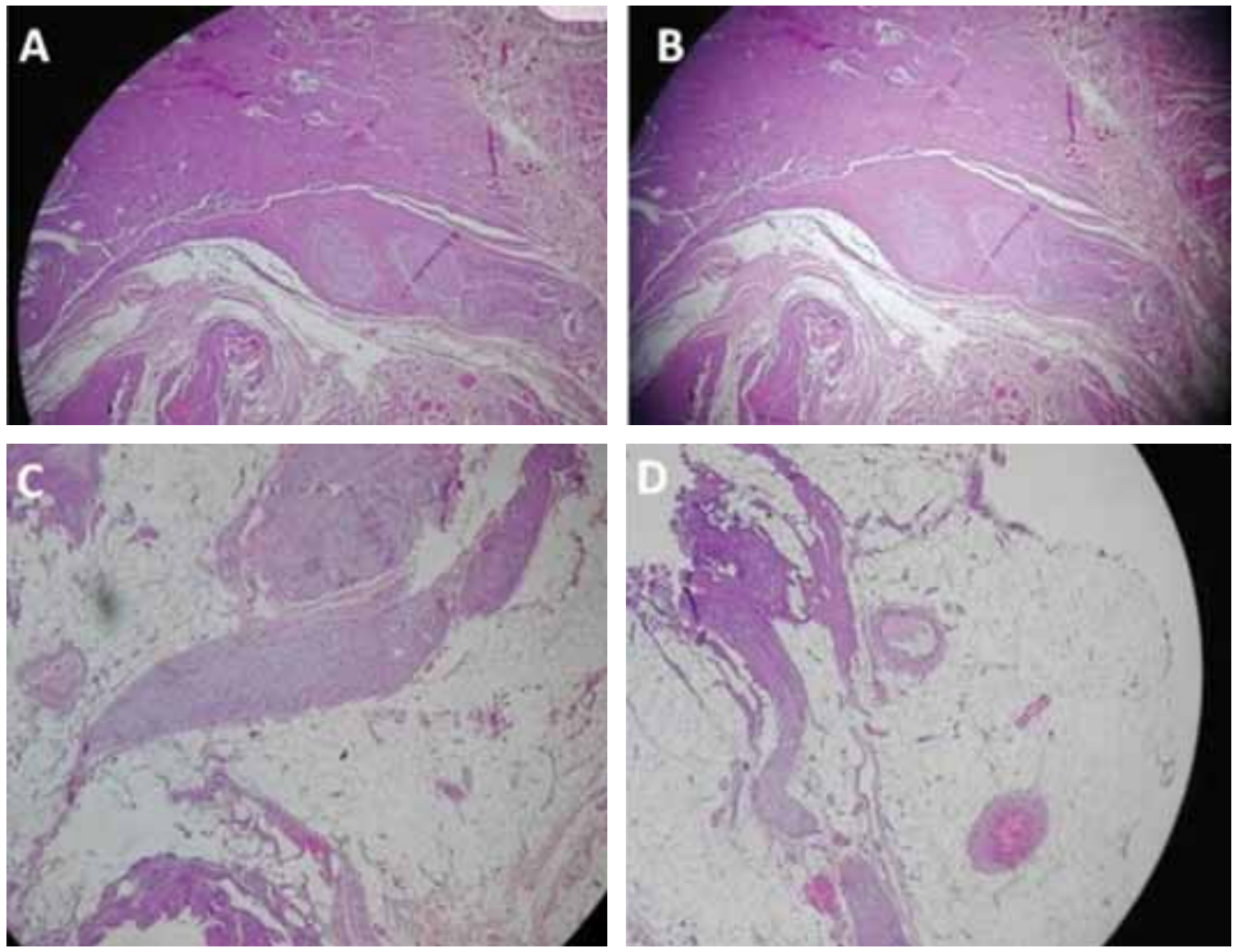

Figure 2. A; B; C; D: Histological analysis: A group of nerve branches surrounded by fibrous tissue and mature fat are located just below the previous mastectomy scar. 

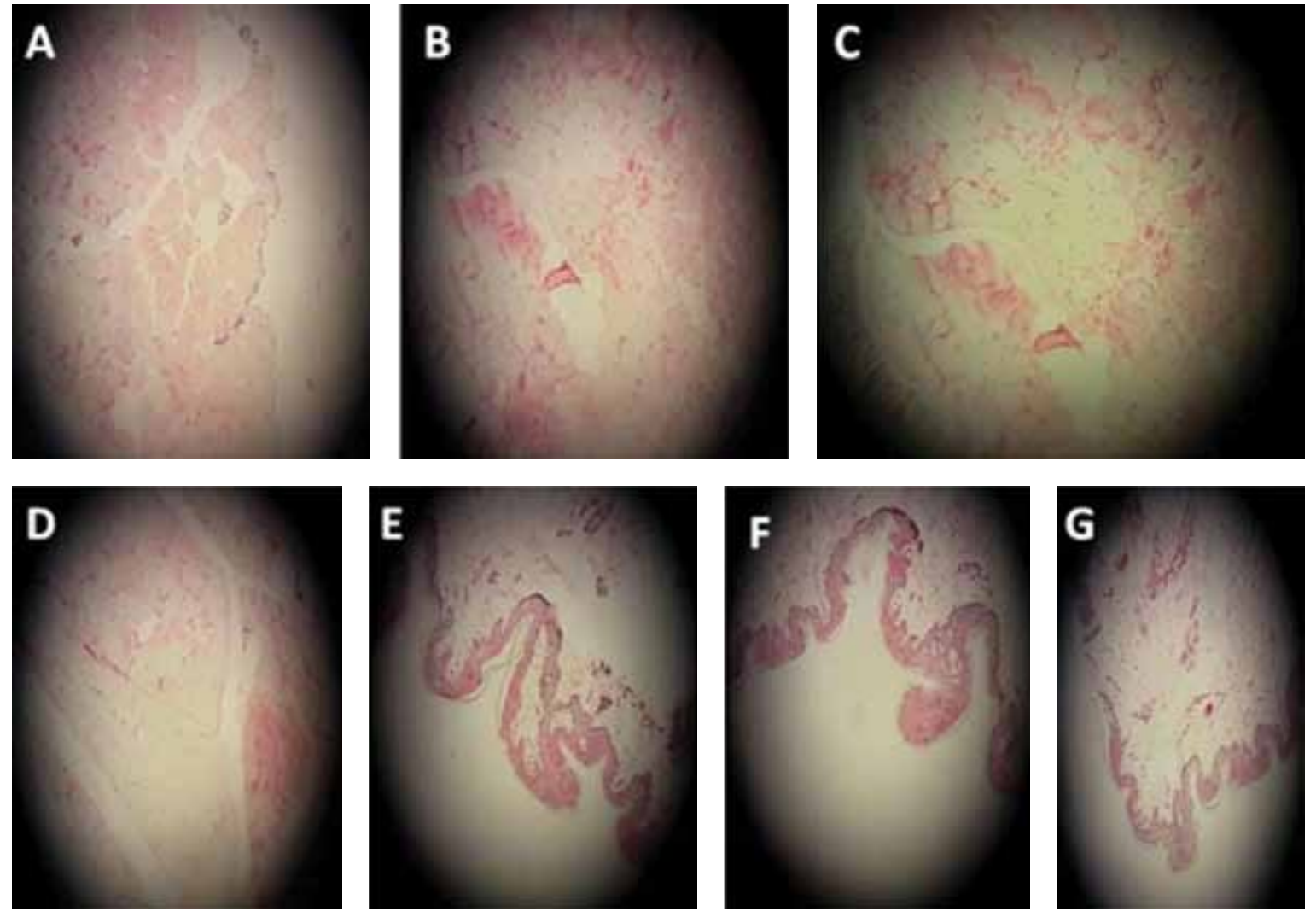

Figure 3. A; B; C; D; E;F;G Immunochemistry application via neuron-specific enolase (nse).

breast surgeries and have to do possible with intersection of nerve branches or grasping them with sutures. On the other hand, removing mastectomy scar minimization of pain or irritation at the mastectomy site is referred. Probably the histological results of the second surgery which showed that nerve branches interfered with muscular and fibrous tissue and the removal of these indicates the reason for breast surgeries pain.

\section{Discussion}

All the surgical procedures have a possibility of complications. Breast surgery major complications are wound infections, seroma, hematoma, comorbidity of the axillary because of nerve intervention, lymphedema and pain ${ }^{4}$. Density of symptoms depends on the type of surgery (involvement of the axillary or not), patient's age, health status, surgical technique and repetitive operation on the same point is done. Pain after a surgical approach remains an important problem for patient's quality of life. It is estimated that affects $20-30 \%$ of patients. Usually is characterized by a sensation of dull, burning and aching feeling, getting worse with movement. Sometimes pain has the character of neuropathic pain with numbness, needle and burning sensation. For many patients who experience a chronic pain for any reason, they seem to have a greater possibility of developing pain after a breast surgery, than in general population. In addition, younger women with an advanced cancer seem to have 
increased pain, since breast cancer develops to younger patient more rapid and they usually have an extensive surgical treatment ${ }^{5}$. On the other hand, persisted pain after unsuccessful treatment for chronic, neuropathic pain should be taken into consideration and the decision for minimal surgery for removing the old scar may should be thought as well. Recently the breast surgery with the detection of sentinel lymph node led to reduction of less minimal breast surgeries. Surgical operation tends to become less traumatic as it necessitates less sutures and diathermy usage, which means less operational time and less nerve and soft tissue trauma of the area. Thus, the incidence of damage of the intercostobrachial nerve is lower, meaning lower post-surgical pain.

\section{Conclusion}

Overall, according to the literature, we did not manage to find a similar case report presenting nerve branches under the mastectomy scar leading to woman's inexplicable pain.

\section{Acknowledgments}

The authors are grateful to all who provided assistance during the preparation of this manuscript.

\section{Conflict of interest statement}

The authors of this paper certify that they have NO affiliations with or involvement in any organization or entity with any financial interest (such as honoraria; educational grants; participation in speakers bureaus; membership, employment, consultancies, stock ownership, or other equity interest; and expert testimony or patent-licensing arrangements), or non-financial interest (such as personal or professional relationships, affiliations, knowledge or beliefs) in the subject matter or materials discussed in this manuscript. This research did not receive any specific grant from funding agencies in the public, commercial, or not-for-profit sectors.

\section{References}

1. Sir Astley PastonCooper, Bart. (http://jdc.jefferson.edu/cgi/viewcontent.cgi?article $=1020 \&$ con text=cooper).

2. Sarhadi NS, Shaw Dunn J, Lee FD, Soutar DS. An anatomical study of the nerve supply of the breast, including the nipple and areola. Br J Plast Surg. 1996 Apr;49(3):156-64.

3. Sarhadi NS, Shaw-Dunn J, Soutar DS. Nerve supply of the breast with special reference to the nipple and areola: Sir Astley Cooper revisited. Clin Anat. 1997;10(4):283-8.

4. Vitug AF, Newman LA. Complications in breast surgery. Surg Clin North Am. 2007 Apr;87(2):431-51

5. Cui L, Fan P, Qiu C, Hong Y. Single institution analysis of incidence and risk factors for postmastectomy pain syndrome. Sci Rep. 2018 Jul 31;8(1):11494.
Received 28-19-2019

Revised 21-11-2019

Accepted 3-12-2019 